\title{
A SPECTRAL APPROACH TO AN INTEGRAL EQUATION
}

\author{
by W. LAMB
}

(Received 5 December, 1983)

1. Introduction. In a recent paper [7], Rooney used a technique involving the Mellin transform to obtain solutions in certain spaces $\mathscr{L}_{\mu, p}$ of an integral equation which had been studied previously by Šub-Sizonenko [9]. The integral equation in question can be written as

$$
\left(I+G^{0.1 / 2}\right) \phi(x)=\psi(x) \quad(x>0),
$$

where $I$ denotes the identity operator and $G^{0.1 / 2}$ is given by

$$
\left(G^{0,1 / 2} \phi\right)(x)=\pi^{-1 / 2} \int_{x}^{\infty}(\log t / x)^{-1 / 2} \phi(t) d t / t,
$$

with the inversion formula obtained by Rooney taking the form

$$
\begin{gathered}
\phi(x)=\int_{x}^{\infty}\left((t / x) \operatorname{erfc}\left((\log t / x)^{1 / 2}\right)-\pi^{-1 / 2}(\log t / x)^{-1 / 2}\right) \psi(t) d t / t \\
+\psi(x) \quad(x>0) .
\end{gathered}
$$

Rooney verified that (1.1) and (1.2) formed an inversion pair in $\mathscr{L}_{\mu, p}$ for $1 \leqslant p<\infty$ and $\mu>0$.

In this paper, we shall extend Rooney's result by obtaining inversion formulae for the integral equations

and

$$
\left(\lambda I+G^{\eta, 1 / 2}\right) \phi(x)=\psi(x) \quad(x>0)
$$

$$
\left(\lambda I+H^{\eta, 1 / 2}\right) \phi(x)=\psi(x) \quad(x>0)
$$

where $\lambda>0$ and $G^{\eta, 1 / 2}$ and $H^{\eta, 1 / 2}$ are particular cases of the operators $G^{\eta, \alpha}$ and $H^{\eta, \alpha}$ defined, for $\operatorname{Re} \alpha>0$ and $\eta \in \mathbb{C}$, by

$$
\begin{array}{ll}
\left(G^{\eta, \alpha} \phi\right)(x)=[\Gamma(\alpha)]^{-1} \int_{x}^{\infty}(x / t)^{\eta}(\log t / x)^{\alpha-1} \phi(t) d t / t & (x>0), \\
\left(H^{\eta, \alpha} \phi\right)(x)=[\Gamma(\alpha)]^{-1} \int_{0}^{x}(t / x)^{\eta+1}(\log x / t)^{\alpha-1} \phi(t) d t / t & (x>0) .
\end{array}
$$

Note that equation (1.3) reduces to equation (1.1) when $\lambda=1$ and $\eta=0$ and therefore in deriving inversion formulae for (1.3) and (1.4), we shall also obtain an inversion formula for (1.1).

Working within the framework of the Banach spaces $L_{\mu}^{p}$ (where $L_{\mu}^{p}=\mathscr{L}_{1 / p-\mu, p}$ when $\mu$ is real), we shall first determine properties of $H^{\eta, \alpha}$ and $G^{\eta, \alpha}$ and shall establish that, under certain conditions, $H^{n_{1} 1 / 2}=\left(H^{n_{1}}\right)^{1 / 2}, G^{\eta_{1} / 2}=\left(G^{n_{1}}\right)^{1 / 2}$ where $\left(H^{n_{1}}\right)^{1 / 2}$ and $\left(G^{n_{1} 1}\right)^{1 / 2}$

Glasgow Math. J. 26 (1985) 83-89. 
denote fractional powers of order $1 / 2$ of $H^{n, 1}$ and $G^{n, 1}$ respectively. Then, by applying a result concerning the resolvent of a fractional power of an operator, we shall obtain the required inversion formulae which will be shown to include (1.2) as a special case.

2. Preliminaries. Let $X$ denote a complex Banach space with norm \|\| and let $L(X)$ denote the Banach space of bounded linear operators $A: X \rightarrow X$. We say that $A$ is in the class $P(X)$ if

(a) $A \in L(X)$;

(b) $R(\lambda ; A) \equiv(\lambda I-A)^{-1} \in L(X)$ for each $\lambda>0$;

(c) $\|\lambda R(\lambda ; A) \phi\| \leqslant M\|\phi\|$ for all $\lambda>0$ and $\phi \in X$ where $M$, a positive constant, is independent of both $\lambda>0$ and $\phi \in X$.

If $A$ is an operator in $P(X)$, then a family of operators $\left\{(-A)^{\alpha} ; \operatorname{Re} \alpha>0\right\}$ can be generated by means of the formulae

$$
\begin{gathered}
(-A)^{\alpha} \phi=\pi^{-1} \sin (\pi \alpha) \int_{0}^{\infty} \lambda^{\alpha-1}\left[R(\lambda ; A)-\lambda /\left(1+\lambda^{2}\right)\right](-A \phi) d \lambda \\
-\sin (\pi \alpha / 2) A \phi, \quad 0<\operatorname{Re} \alpha<2, \quad \phi \in X, \\
(-A)^{\alpha} \phi=(-A)^{\alpha-n}(-A)^{n} \phi, \quad n<\operatorname{Re} \alpha<n+2, \quad n=1,2, \ldots, \phi \in X .
\end{gathered}
$$

By appealing to conditions (b) and (c) above, we can readily show that the integral in (2.1) exists, for each $\phi \in X$, as a Bochner integral in $X$ (see [4, p. 34 and pp. 118-119]). The main properties of the operators $(-A)^{\alpha}$ are summarised below.

TheOREM 2.1. Let $A$ be an operator in $P(X)$ and let $(-A)^{\alpha}$ be defined via (2.1) and (2.2). Then

(a) $(-A)^{\alpha} \in L(X)$ for each $\alpha$ such that $\operatorname{Re} \alpha>0$;

(b) $(-A)^{\alpha}(-A)^{\beta}=(-A)^{\alpha+\beta}$ for $\operatorname{Re} \alpha, \operatorname{Re} \beta>0$;

(c) $\left[(-A)^{\alpha}\right]^{\beta}=(-A)^{\alpha \beta}$ for $0<\alpha<1$ and $\operatorname{Re} \beta>0$;

(d) for each $\lambda>0$ and $\alpha \in(0,1)$, the resolvent operator $R\left(\lambda ;-(-A)^{\alpha}\right)$ exists in $L(X)$ and is given by the Bochner integral

$$
R\left(\lambda ;-(-A)^{\alpha}\right) \phi=\int_{0}^{\infty} g_{\lambda, \alpha}(u) R(u ; A) \phi d u \quad(\phi \in X)
$$

where

$$
g_{\lambda, \alpha}(u)=\pi^{-1} \sin (\pi \alpha) u^{\alpha}\left[\lambda^{2}+2 \lambda u^{\alpha} \cos (\pi \alpha)+u^{2 \alpha}\right]^{-1}
$$

Proof. These results can be found in [1] and [3] and can also be deduced as a special case of the theory presented in [4] and [5].

Motivated by the properties possessed by the operators $(-A)^{\alpha}$, we shall henceforth refer to $(-A)^{\alpha}$ as the $\alpha$ th power of $-A$.

3. The operators $G^{\eta, \alpha}$ and $H^{\eta, \alpha}$ on the spaces $L_{\mu}^{p}$. In this section, we shall determine certain properties of the integral operators $G^{\eta, \alpha}$ and $H^{\eta, \alpha}$ given by (1.5) and (1.6) respectively. In particular, we shall examine the behaviour of these operators on the 
spaces $L_{\mu}^{p}$ of (equivalence classes of) functions $\phi$ such that $\int_{0}^{\infty}\left|x^{-\mu} \phi(x)\right|^{p} d x<\infty$. Here, and in the sequel, $\mu$ is any complex number and $1 \leqslant p<\infty$. Equipped with the norm \|\|$_{p, \mu}$ defined by

$$
\|\phi\|_{p, \mu}=\left(\int_{0}^{\infty}\left|x^{-\mu} \phi(x)\right|^{p} d x\right)^{1 / p}
$$

the space $L_{\mu}^{p}$ is a Banach space and, for $\mu$ real, coincides with the space $\mathscr{L}_{1 / \mathrm{p}-\mu, \mathrm{p}}$ of Rooney $[\mathbf{8}]$.

Lemma 3.1. Let $\operatorname{Re} \alpha>0$ and $\phi \in L_{\mu}^{p}$.

(a) If $\operatorname{Re}(\eta+\mu)+1>1 / p$, then $H^{\eta, \alpha}$ is a bounded operator on $L_{\mu}^{p}$ with

$$
\left.\left\|H^{\eta, \alpha} \phi\right\|_{p, \mu} \leqslant(\Gamma(\operatorname{Re} \alpha) / \mid \Gamma(\alpha))\right)(\operatorname{Re}(\eta+\mu)+1-1 / p)^{-\operatorname{Re} \alpha}\|\phi\|_{p, \mu} .
$$

(b) If $\operatorname{Re}(\eta-\mu)>-1 / p$, then $G^{\eta, \alpha}$ is a bounded operator on $L_{\mu}^{p}$ with

$$
\left\|G^{n, \alpha} \phi\right\|_{p, \mu} \leqslant(\Gamma(\operatorname{Re} \alpha) /|\Gamma(\alpha)|)(\operatorname{Re}(\eta-\mu)+1 / p)^{-\operatorname{Re} \alpha}\|\phi\|_{p, \mu} .
$$

Proof. This can be proved in a routine manner by using a generalisation of an inequality of Hardy [6].

THEOREM 3.2. Let $\operatorname{Re} \alpha>0$.

(a) If $\operatorname{Re}(\eta+\mu)+1>1 / p$, then $-H^{\eta, 1} \in P\left(L_{\mu}^{p}\right)$ and $\left(H^{\eta, 1}\right)^{\alpha}=H^{\eta, \alpha}$.

(b) If $\operatorname{Re}(\eta-\mu)>-1 / p$, then $-G^{\eta, 1} \in P\left(L_{\mu}^{p}\right)$ and $\left(G^{\eta, 1}\right)^{\alpha}=G^{\eta, \alpha}$.

Proof. We shall prove (a), the proof of (b) being similar. Firstly, we remark that, under the given conditions, $-H^{\eta, 1} \in L\left(L_{\mu}^{p}\right)$. Secondly, a routine calculation (see [4, p. 67)]) can be used to show that, for $\lambda>0$,

$$
\left(\lambda I+H^{\eta, 1}\right)^{-1} \phi=(1 / \lambda) \phi-(1 / \lambda)^{2} H^{\eta+1 / \lambda, 1} \phi, \quad \phi \in L_{\mu}^{p} .
$$

Hence, it follows that

$$
\begin{aligned}
\| \lambda(\lambda I & \left.+H^{\eta .1}\right)^{-1} \phi \|_{p, \mu} \\
& =\left\|\phi-(1 / \lambda) H^{\eta+1 / \lambda, 1} \phi\right\|_{p, \mu} \\
& \leqslant\|\phi\|_{p, \mu}+(1 / \lambda)(\operatorname{Re}(\eta+\mu+1 / \lambda)+1-1 / p)^{-1}\|\phi\|_{p, \mu} \quad \text { (from (3.1)) } \\
& <2\|\phi\|_{p, \mu}
\end{aligned}
$$

and this holds for all $\lambda>0$. Consequently, $-H^{\eta, 1}$ belongs to the class $P\left(L_{\mu}^{p}\right)$ and so, from Theorem 2.1(a), $\left(H^{\eta, 1}\right)^{\alpha}$ exists as a bounded operator on $L_{\mu}^{p}$. Now let $\psi$ belong to the space $C_{0}^{\infty}(0, \infty)$ of smooth functions having compact support in $(0, \infty)$ and suppose that $0<\operatorname{Re} \alpha<1$. In this case, formula (2.1) can be replaced by

$$
(-A)^{\alpha} \phi=\pi^{-1} \sin (\pi \alpha) \int_{0}^{\infty} \lambda^{\alpha-1} R(\lambda ; A)(-A) \phi d \lambda \quad(\text { see }[1] \text { and }[5]),
$$


and therefore

$$
\begin{aligned}
\left(H^{\eta, 1}\right)^{\alpha} \psi(x) & =\left(\pi^{-1} \sin (\pi \alpha) \int_{0}^{\infty} \lambda^{\alpha-2} H^{n+1 / \lambda, 1} \psi d \lambda\right)(x) \quad(x>0) \\
& =\pi^{-1} \sin (\pi \alpha) \int_{0}^{\infty} \lambda^{\alpha-2} H^{n+1 / \lambda, 1} \psi(x) d \lambda \\
& =\pi^{-1} \sin (\pi \alpha) \int_{0}^{\infty} \lambda^{\alpha-2} \int_{0}^{x}(t / x)^{n+1 / \lambda+1} \psi(t) d t / t d \lambda
\end{aligned}
$$

The justification for transferring the point $x$ inside the Bochner integral in the above analysis is provided by [4, Theorems 4.19 and 4.24]. If we now apply Fubini's theorem to interchange the order of integration, we obtain

$$
\begin{aligned}
\left(H^{\eta, 1}\right)^{\alpha} \psi(x) & =\pi^{-1} \sin (\pi \alpha) \int_{0}^{x}(t / x)^{\eta+1} \psi(t) d t / t \int_{0}^{\infty} \lambda^{\alpha-2} \exp \left[-\lambda^{-1} \log (x / t)\right] d \lambda \\
& =[\Gamma(\alpha)]^{-1} \int_{0}^{x}(t / x)^{\eta+1}(\log x / t)^{\alpha-1} \psi(t) d t / t \\
& =\left(H^{\eta, \alpha} \psi\right)(x) .
\end{aligned}
$$

In a similar fashion, we can prove that $\left(H^{\eta, 1}\right)^{\alpha} \psi=H^{\eta, \alpha} \psi$ for $n<\operatorname{Re} \alpha<n+1, n=$ $1,2, \ldots$, and $\psi \in C_{0}^{\infty}(0, \infty)$ while, for $\alpha=n+i \xi$, we have

$$
\begin{aligned}
\left(H^{n, 1}\right)^{n+i \xi} \psi & =\left[\left(H^{\eta, 1}\right)^{(n+i \xi) / 2 n}\right]^{2 n} \psi \\
& =\left[H^{n,(n+i \xi) / 2 n}\right]^{2 n} \psi \\
& =H^{\eta, n+i \xi} \psi
\end{aligned}
$$

where the last step can be verified by direct calculation. This proves that $\left(H^{\eta, 1}\right)^{\alpha}=H^{\eta, \alpha}$, $\operatorname{Re} \alpha>0$, as operators on $C_{0}^{\infty}(0, \infty)$ and the general result follows from the continuity of the operators on $L_{\mu}^{p}$ in conjunction with the denseness of $C_{0}^{\infty}(0, \infty)$ in $L_{\mu}^{p}$. This completes the proof.

Using the properties of fractional powers listed in Theorem 2.1, we can now write down the properties of $H^{\eta, \alpha}$ and $G^{n, \alpha}$ on $L_{\mu}^{p}$.

TheOREM 3.3. Let $\operatorname{Re}(\eta+\mu)+1>1 / p$ and $\phi \in L_{\mu}^{p}$. Then

(a) $H^{\eta, \alpha} H^{\eta, \beta} \phi=H^{\eta, \alpha+\beta} \phi$ for $\operatorname{Re} \alpha, \operatorname{Re} \beta>0$;

(b) $\left(H^{\eta, \alpha}\right)^{\beta} \phi=H^{\eta, \alpha \beta} \phi \quad$ for $0<\alpha<1$ and $\operatorname{Re} \beta>0$.

THEOREM 3.4. Let $\operatorname{Re}(\eta-\mu)>-1 / p$ and $\phi \in L_{\mu}^{p}$. Then

(a) $G^{\eta, \alpha} G^{\eta, \beta} \phi=G^{\eta, \alpha+\beta} \phi$ for $\operatorname{Re} \alpha, \operatorname{Re} \beta>0$;

(b) $\left(G^{\eta, \alpha}\right)^{\beta} \phi=G^{\eta, \alpha \beta} \phi \quad$ for $0<\alpha<1, \operatorname{Re} \beta>0$.

4. The resolvent operators $R\left(\lambda ;-G^{\eta, 1 / 2}\right)$ and $R\left(\lambda ;-H^{\eta, 1 / 2}\right)$. In this final section, we apply the results of Sections 2 and 3 to obtain solutions of equations (1.3) and (1.4) 
when the right-hand side $\psi$ belongs to $L_{\mu}^{p}$. We begin by stating the following results concerning the resolvent operators $R\left(\lambda ;-G^{\eta, \alpha}\right)$ and $R\left(\lambda ;-H^{\eta, \alpha}\right)$. Then

THEOREM 4.1. Let $\lambda>0,0<\alpha<1, \eta \in \mathbb{C}$ and let $g_{\lambda, \alpha}$ be the function defined by (2.4).

(a) if $\operatorname{Re}(\eta+\mu)+1>1 / p$, the resolvent operator $R\left(\lambda ;-H^{\eta, \alpha}\right)$ exists in $L\left(L_{\mu}^{p}\right)$ and is given by

$$
R\left(\lambda ;-H^{\eta, \alpha}\right) \phi=(1 / \lambda) \phi-\int_{0}^{\infty} g_{\lambda, \alpha}(u) u^{-2} H^{\eta+1 / u, 1} \phi d u \quad\left(\phi \in L_{\mu}^{p}\right)
$$

(b) if $\operatorname{Re}(\eta-\mu)>-1 / p$, the resolvent operator $R\left(\lambda ;-G^{\eta, \alpha}\right)$ exists in $L\left(L_{\mu}^{p}\right)$ and is given by

$$
R\left(\lambda ;-G^{\eta, \alpha}\right) \phi=(1 / \lambda) \phi-\int_{0}^{\infty} g_{\lambda, \alpha}(u) u^{-2} G^{\eta+1 / u, 1} \phi d u \quad\left(\phi \in L_{\mu}^{p}\right)
$$

The integrals which appear in (4.1) and (4.2) exist as Bochner integrals in $L_{\mu}^{p}$.

Proof. Formula (4.1) follows immediately from Theorem 2.1(d), (3.3) and the fact that $\int_{0}^{\infty} u^{-1} g_{\lambda, \alpha}(u) d u=\lambda^{-1}$ for $0<\alpha<1$ and $\lambda>0$ (see [3]). The derivation of (4.2) is similar.

From Theorem 4.1, we can deduce immediately that the equations

$$
\left(\lambda I+H^{\eta, \alpha}\right) \phi=\psi ;\left(\lambda I+G^{\eta, \alpha}\right) \phi=\psi, \quad\left(\psi \in L_{\mu}^{p}, 0<\alpha<1, \lambda>0\right)
$$

have unique solutions in $L_{\mu}^{p}$ under appropriate restrictions on $\eta, \mu$ and $p$. For the particular case when $\alpha=1 / 2$ we can proceed as follows to determine these solutions more explicitly.

THEOREM 4.2. Let $\lambda>0$ and $\zeta \in L_{\mu}^{p}$.

(a) If $\operatorname{Re}(\eta+\mu)+1>1 / p$, then

$$
\begin{aligned}
& \left(\lambda I+H^{\eta, 1 / 2}\right)^{-1} \zeta(x) \\
& \quad=(1 / \lambda) \zeta(x)-\left(\lambda^{3} \pi\right)^{-1} \Gamma(3 / 2) \int_{0}^{x} \Psi\left(3 / 2 ; 3 / 2 ; \lambda^{-2} \log x / t\right)(t / x)^{\eta+1} \zeta(t) d t / t \quad(x>0),
\end{aligned}
$$

where $\Psi$ is as defined in [2, p. 255].

(b) If $\operatorname{Re}(\eta-\mu)>-1 / p$, then

$$
\begin{aligned}
(\lambda I & \left.+G^{\eta, 1 / 2}\right)^{-1} \zeta(x) \\
& =(1 / \lambda) \zeta(x)-\left(\lambda^{3} \pi\right)^{-1} \Gamma(3 / 2) \int_{x}^{\infty} \Psi\left(3 / 2 ; 3 / 2 ; \lambda^{-2} \log t / x\right)(x / t)^{n} \zeta(t) d t / t \quad(x>0) .
\end{aligned}
$$

Proof. (a). Let $T$ be the operator defined on $L_{\mu}^{p}$ by

$$
T \zeta=\int_{0}^{\infty} u^{-2} g_{\lambda, 1 / 2}(u) H^{\eta+1 / u, 1} \zeta d u
$$


By proceeding as in the proof of Theorem 3.2, we can verify that, for each $x>0$,

$$
\begin{aligned}
(T \zeta)(x) & =\int_{0}^{\infty} u^{-2} g_{\lambda, 1 / 2}(u) H^{\eta+1 / u, 1} \zeta(x) d u \\
& =\int_{0}^{\infty} u^{-2} g_{\lambda, 1 / 2}(u) \int_{0}^{x}(t / x)^{n+1 / u+1} \zeta(t) d t / t d u \\
& =\int_{0}^{x}(t / x)^{\eta+1} \zeta(t) d t / t \int_{0}^{\infty}(t / x)^{1 / u} u^{-2} g_{\lambda, 1 / 2}(u) d u \quad \text { (by Fubini's theorem) }
\end{aligned}
$$

provided $\zeta \in C_{0}^{\infty}(0, \infty)$. The inner integral can be evaluated using [2, p. 255(2)] and we can state that, for $\zeta \in C_{0}^{\infty}(0, \infty)$,

$$
(T \zeta)(x)=\left(\lambda^{3} \pi\right)^{-1} \Gamma(3 / 2) \int_{0}^{x}(t / x)^{\eta+1} \Psi\left(3 / 2 ; 3 / 2 ; \lambda^{-2} \log x / t\right) \zeta(t) d t / t .
$$

If we now apply the extended version of Hardy's inequality [6] in conjunction with the asymptotic expansion for $\Psi$ given in $[2$, p. 278(1)], we can deduce that the operator defined by the right-hand side of (4.5) is in $L\left(L_{\mu}^{p}\right)$ under the stated conditions on $\eta, \mu$ and p. Consequently, from (4.1), the continuity of the operators and the denseness of $C_{0}^{\infty}(0, \infty)$ in $L_{\mu}^{p}$, it follows that $\left(\lambda I+H^{\eta, 1 / 2}\right)^{-1} \zeta(x)$ is given by (4.3) for any $\zeta \in L_{\mu}^{p}$. This completes the proof of (a). The proof of (b) is similar.

COROLlary 4.3. Under the conditions stated in Theorem 4.2, we can write

$$
\begin{aligned}
\left(\lambda I+H^{\eta, 1 / 2}\right)^{-1} \zeta(x)= & (1 / \lambda) \zeta(x)-\left(\lambda^{2} \Gamma(1 / 2)\right)^{-1} \int_{0}^{x}(\log x / t)^{-1 / 2}(t / x)^{\eta+1} \zeta(t) d t / t \\
& +(1 / \lambda)^{3} \int_{0}^{x}(t / x)^{\eta+1-1 / \lambda^{2}} \operatorname{erfc}\left[\lambda^{-1}(\log x / t)^{1 / 2}\right] \zeta(t) d t / t \\
\left(\lambda I+G^{\eta, 1 / 2}\right)^{-1} \zeta(x)= & (1 / \lambda) \zeta(x)-\left(\lambda^{2} \Gamma(1 / 2)\right)^{-1} \int_{x}^{\infty}(\log t / x)^{-1 / 2}(x / t)^{\eta} \zeta(t) d t / t \\
& +(1 / \lambda)^{3} \int_{x}^{\infty}(x / t)^{\eta-1 / \lambda^{2}} \operatorname{erfc}\left[\lambda^{-1}(\log t / x)^{1 / 2}\right] \zeta(t) d t / t .
\end{aligned}
$$

Proof. We note first that the function $\Psi(3 / 2 ; 3 / 2 ; x)$ can be written as $e^{x} \Gamma(-1 / 2 ; x)$ $[2$, p. $266(21)]$ which in turn can be expressed as $-2 e^{x}\left(\Gamma(1 / 2 ; x)-x^{-1 / 2} e^{-x}\right)$.

Since $\Gamma(1 / 2 ; x)=\sqrt{\pi} \operatorname{erfc}(\sqrt{x})[2$, p. 266(24)], substitution into (4.3) and (4.4) gives the stated formulae.

Finally, if we set $\eta=0$ and $\lambda=1$, then, from Theorem 4.2 and Corollary 4.3 , we can state that (1.1) has a unique solution in $L_{\mu}^{p}$, given by (1.2), whenever $\psi \in L_{\mu}^{p}$ and $\operatorname{Re} \mu<1 / p$. This agrees with the result obtained by Rooney.

\section{REFERENCES}

1. A. V. Balakrishnan, Fractional powers of closed operators and semigroups generated by them, Pacific J. Math. 10 (1960), 419-437. 
2. A. Erdélyi et al., Higher transcendental functions I (McGraw-Hill, 1953).

3. H. W. Hövel and U. Westphal, Fractional powers of closed operators, Studia Math. 42 (1972), 177-194.

4. W. Lamb, Fractional powers of operators on Fréchet spaces with applications (Strathclyde Univ. Ph.D. Thesis, 1980).

5. W. Lamb, Fractional powers of operators defined on a Fréchet space, Proc. Edinburgh Math. Soc. (2), (to appear).

6. E. R. Love, Some inequalities for fractional integrals, Linear spaces and approximation, Proc. Conf., Math. Res. Int. Oberwolfach, 1977, Internat. Ser. Numer. Math., 40 (Birkhauser, 1978).

7. P. G. Rooney, On an integral equation of Sub-Sizonenko, Glasgow Math. J. 24 (1983), 207-210.

8. P. G. Rooney, Multipliers for the Mellin transformation, Canad. Math. Bull. 25 (1982), 257-262.

9. J. A. Sub-Sizonenko, Inversion of an integral operator by the method of expansion with respect to orthogonal Watson operators, Siberian Math. J. 20 (1979), 318-321.

Department of Mathematics

UNIVERSITY OF STRATHCLYDE

GLASGOW 